\title{
Flux Exclusion Quantum Superconducting Metamaterial
}

\author{
V. Savinov ${ }^{1, *}$, A. Tsiatmas ${ }^{1}$, A. R. Buckingham ${ }^{2}$, V. A. Fedotov ${ }^{1}$, P. A. J. $\operatorname{de~Groot~}^{2}$ and N. I. Zheludev ${ }^{1}$ \\ ${ }^{1}$ Optoelectronics Research Centre \& Centre for Photonic Metamaterials, University of Southampton, SO17 1BJ, UK \\ ${ }^{2}$ School of Physics and Astronomy \& Centre for Photonic Metamaterials, University of Southampton, SO17 1BJ, UK \\ *vs1106@orc.soton.ac.uk
}

\begin{abstract}
The new type of metamaterial exploits magnetic flux quantization as a source of its nonlinear response but does not require Josephson junctions. We fabricated metamaterial from a high- $\mathrm{T}_{\mathrm{c}}$ superconductor and report its electromagnetic characterization. OCIS codes: (160.3918) Metamaterials; (160.4330) Nonlinear optical materials;
\end{abstract}

We introduce a new type of superconductor metamaterial with quantum level nonlinearity that does not require Josephson junctions. Metamaterial is manufactured from a high- $\mathrm{T}_{\mathrm{c}}$ superconductor Yttrium-Barium-CopperOxide (YBCO). Its electromagnetic properties are characterized as a function of frequency and temperature by experimentally modelling the low and high intensity regimes. Sharp switching behaviour between modelled low and high intensity quantum regimes is observed.

To engage the flux quantization mechanism as a source of nonlinearity in the metamaterial we enclose a nest of superconducting wire loops into a resonant split-ring resonator thus creating a meta-molecule, the building block of metamaterial that resembles a wood-cut. For an electromagnetic wave normally incident on the array of such meta-molecules, the magnetic field of the incident wave is not directly engaged in the flux quantization. However, incident electromagnetic wave polarized along the gap will drive an oscillating current in the loop that will produce oscillating magnetic field embraced by the split-ring. The nest of superconducting wire loops inside the split-ring will respond depending on the amplitude of the generated magnetic field: it will block the field penetration inside the ring at low levels of magnetic field, or will allow penetration at high levels of excitation. This will modify the inductive properties of the meta-molecule and thus its resonant response. The profile microscope picture of the metamaterial is shown on Fig. 1.

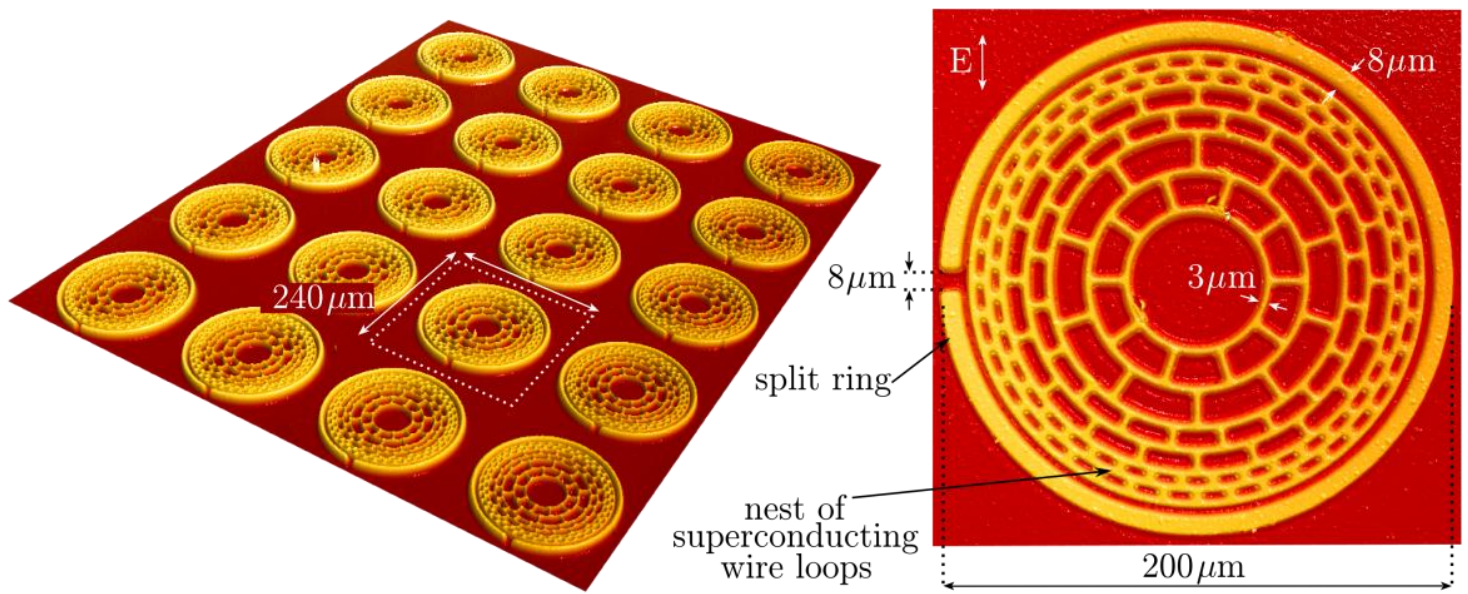

Fig 1: Wood-cut flux exclusion superconducting quantum metamaterial. The microscope profile scan shows a fragment of the fabricated metamaterial array (colour expresses height) and a single wood-cut meta-molecule. The metamaterial is created by patterning 300nm-thick YBCO film deposited onto $1 \mathrm{~mm}$-thick sapphire substrate. The unit cell size is $240 \mu \mathrm{m} \times 240 \mu \mathrm{m}$.

The metamaterial optimized to operate at the resonance frequency of about $100 \mathrm{GHz}$ was manufactured from YBCO on a sapphire substrate. Metamaterial has been characterized in the closed-cycle helium-cooled cryostat (see Fig. 2a) using a sub-terahertz network analyser.

Fig $2 b$ shows experimentally measured transmission of the new quantum exclusion metamaterial for a low and high intensity regime. Here high intensity regime is modelled by removing the wood-cur section from the split ring. We estimate the required intensity for observation of nonlinear effects to be of order $1 \mathrm{~W} / \mathrm{cm}^{2}$. All measurements are taken at intensity level of about $100 \mu \mathrm{W} / \mathrm{cm}^{2}$. In good agreement with full 3D Maxwell simulations, the experimental results show a clear LC-like resonance at $95 \mathrm{GHz}$ that shifts down in frequency as temperature is increased A considerable change of transmission upon switching from the field exclusion regime (low intensity) to field penetration regime (high intensity) is observed. 

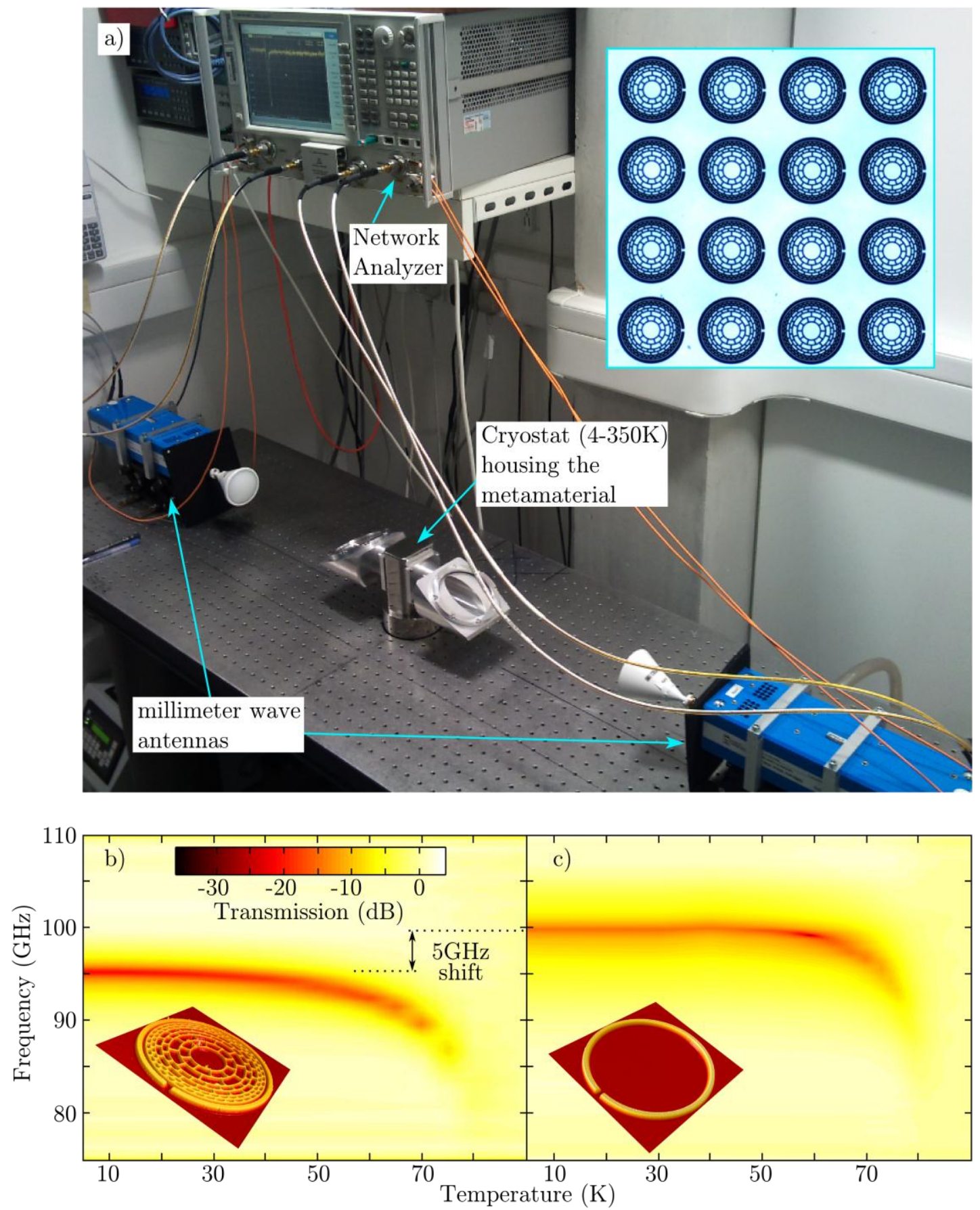

Fig 2: Electromagnetic response of the wood-cut metamaterial (low and high intensity regimes). a) The millimetre wave experimental setup. Network Analyzer drives the millimetre wave antennas. The generated radiation penetrates into the closed-cycle helium-cooled cryostat through the transparent windows which allows to measure both reflection and transmission of the superconducting metamaterial housed inside the cryostat; The inset shows an optical microscope picture of the wood-cut metamaterial sample. b) Low intensity transmission of the wood-cut metamaterial as a function of frequency and temperature. The inset shows a profile microscope picture of the meta-atom. c) High intensity transmission of the wood-cut metamaterial as modelled by the split ring metamaterial. The inset shows a profile microscope picture of the unit cell of the split ring metamaterial. Both (b) and (c) share the same scale represented by colourbar in (b).

In conclusion, we proposed and characterized a new type of nonlinear metamaterial targeting microwave and terahertz spectrum. Sharp switching behaviour between modelled low and high intensity quantum regimes is observed. 